\title{
A Finite Element Method for First Order Hyperbolic Equations*
}

\author{
By Garth A. Baker
}

\begin{abstract}
A class of finite element methods is proposed for first order hyperbolic equations. The expository example chosen is of a single equation in one space dimension with constant coefficients.

Optimal $L^{2}$ error estimates are derived for both approximations continuous in the time variable and an approximation scheme discrete in time.
\end{abstract}

1. Introduction. In this work, two finite element schemes for a mixed initialvalue boundary value problem for a single first order hyperbolic equation in one space dimension are proposed, using a nonstandard variational formulation.

The results are obtainable for the corresponding Cauchy problem and for more general first order hyperbolic systems. The simple example chosen here is for ease of exposition.

The schemes fall in analogy with the so-called $H^{-1}$ Galerkin methods proposed by Rachford and Wheeler [5] for two point boundary value problems.

In this work, the Galerkin approximation is obtained by the use of specially chosen spaces of trial functions and test functions in a weak-weak formulation of the boundary value problem. The above spaces are chosen to be compatible with this variational formulation.

The first scheme produces an approximation continuous in the time variable with optimal $L^{2}$ error estimates of $O\left(h^{r}\right)$, where the solution is approximated in a space of discontinuous (nonconforming) piecewise polynomial functions of degree $r-1, r \geqslant 1$.

Secondly, a Crank-Nicolson type time discretization yields approximations discrete in time with again optimal $L^{2}$ error estimates of $O\left(h^{r}+\tau^{2}\right)$, using the above space of functions: $\tau$ denotes the discrete time step. The method is unconditionally convergent and stable.

\section{Notation.}

2.1. Function Spaces. Let $\Omega=(0,1)$, and let $0<T<\infty$ be fixed. For $s \geqslant 0$, $H^{s}(\Omega)$ will denote the Sobolev space $W_{2}^{s}(\Omega)$ of real-valued functions on $\Omega$, and $\|\cdot\|_{s}$ will denote the corresponding norm.

For definitions and the relevant properties of these spaces, see for example [4] . For $s \leqslant 0$ the spaces $H^{s}(\Omega)$ are defined following [6], as the completion of $C^{\infty}(\Omega)$, the set of infinitely differentiable functions on $\Omega$, with respect to the norm:

$$
\|v\|_{s}=\operatorname{SUP}_{\psi \in C^{\infty}(\Omega)} \frac{\left|\int_{0}^{1} v \psi d x\right|}{\|\psi\|_{-s}}, \quad v \in C^{\infty}(\Omega) .
$$

Received April 15, 1975.

AMS (MOS) subject classifications (1970). Primary 65N30.

${ }^{*}$ This research was supported by National Science Foundation Grant GP-34723. 
For $v$ a mapping $v:[0, T] \rightarrow H^{s}(\Omega)$, the following norms are defined,

$$
\|\|_{v} \|_{2, s}=\left(\int_{0}^{T}\|v(\cdot, t)\|_{s}^{2}\right)^{1 / 2}
$$

and

$$
\|\| v\left\|_{\infty, s}=\underset{0 \leqslant t \leqslant T}{\operatorname{SUP}}\right\| v(\cdot, t) \|_{s} .
$$

Following [4], we also adopt the notation

$$
L^{p}\left(0, T ; H^{s}(\Omega)\right)=\left\{v:[0, T] \rightarrow H^{s}(\Omega):\|\| v \|_{p, s}<\infty\right\}, \quad p=2, \infty .
$$

We note also that $H^{0}(\Omega)=L^{2}(\Omega)$, the Hilbert space of real-valued functions square integrable with respect to Lebesgue measure on $\Omega$. We shall write

$$
(u, v)=\int_{0}^{1} u v d x, \quad u, v \in L^{2}(\Omega)
$$

Also, we define the space

$$
\stackrel{\circ}{H}(\Omega)=\left\{v \in H^{1}(\Omega): v(1)=0\right\} .
$$

2.2. The Boundary Value Problem. We shall be interested in approximating the solution of the following mixed initial-value boundary value problem. A function $u: \Omega \times[0, T] \rightarrow R$ is sought satisfying

$$
\left\{\begin{array}{l}
\frac{\partial u}{\partial t}(x, t)+\frac{\partial u}{\partial x}(x, t)=f(x, t), \quad(x, t) \in \Omega \times(0, T] \\
u(0, t)=g(t), \quad t>0 \\
u(x, 0)=u_{0}(x), \quad x \in \Omega .
\end{array}\right.
$$

The functions $f, g$ and $u_{0}$ are given. Henceforth it will be assumed that $f, g$ and $u_{0}$ are such that a unique solution $u$ exists, for $t \geqslant 0$. In the appropriate places to follow, precise conditions on the smoothness of $u$ sufficient to guarantee the convergence results will be imposed.

Define the following bilinear form $b(\cdot, \cdot): L^{2}(\Omega) \times H^{1}(\Omega) \rightarrow R^{1}$ by

$$
b(u, v)=-(u, \partial v / \partial x) \text {. }
$$

Observe that the boundary value problem (2.1) has the following weak-weak formulation: $u \in L^{2}\left(0, T ; L^{2}(\Omega)\right)$ satisfies

$$
((\partial u / \partial t)(\cdot, t), v)+b(u(\cdot, t), v)=(f(\cdot, t), v)+g(t) v(0)
$$

for all $v \in \stackrel{\circ}{H}(\Omega), t>0$.

2.3. The Finite-Dimensional Function Spaces. Let $\Pi(\Omega)$ denote the set of partitions $\Delta$ of $\Omega$, of the form

$$
\Delta: 0=x_{0}<x_{1}<\cdots<x_{N}=1 \text { for } N \geqslant 1 .
$$

Given $\Delta \in \Pi(\Omega)$, set $h=\max _{1 \leqslant i \leqslant N} x_{i}-x_{i-1}$.

For $D \subset \Omega$ and integer $r \geqslant 0, P_{r}(D)$ will denote the set of polynomials of degree 
$\leqslant r$ on $D$. We now define certain finite-dimensional function spaces relative to $\Delta$, which will be used to obtain the approximations.

For $r \geqslant 1$, define

$$
G_{\Delta}^{r}(\Omega)=\left\{v: v \in P_{r-1}\left(x_{i-1}, x_{i}\right), i=1,2, \ldots, N\right\},
$$

and

$$
\stackrel{\circ}{H}_{\Delta}^{r}(\Omega)=\left\{v \in C(\Omega): v \in P_{r}\left(x_{i-1}, x_{i}\right), i=1,2, \ldots, N, v(1)=0\right\}
$$

It is easily see that $G_{\Delta}^{r}(\Omega)$ is a subspace of $L^{2}(\Omega)$, that $\stackrel{\circ}{H}_{\Delta}^{r}(\Omega)$ is a subspace of $\stackrel{\circ}{H}(\Omega)$, and that

$$
\text { Dimension } G_{\Delta}^{r}(\Omega)=\text { Dimension } \stackrel{\circ}{H}_{\Delta}^{r}(\Omega)=N r \text {. }
$$

Let $\left\{\alpha_{1}, \alpha_{2}, \ldots, \alpha_{d}\right\}$ be a basis for $G_{\Delta}^{r}(\Omega)$, and let $\left\{\beta_{1}, \beta_{2}, \ldots, \beta_{d}\right\}$ be a basis for $\stackrel{\circ}{H}_{\Delta}^{r}(\Omega)$, where $d=d(r, \Delta)=N r$.

The following simple result will be needed.

Lemma 2.1. The matrix $G=\left(\left(\alpha_{j}, \beta_{i}\right)\right)_{i, j=1,2, \ldots, d}$ is nonsingular.

Proof. We show that if $\hat{u}=\left(\hat{u}_{1}, \hat{u}_{2}, \ldots, \hat{u}_{d}\right) \in R^{d}$, with $\hat{u} \neq 0$, then there exists a $\hat{v}=\left(\hat{v}_{1}, \hat{v}_{2}, \ldots, \hat{v}_{d}\right)^{T} \in R^{d}$ such that $\hat{v}^{T} G \hat{u}>0$.

To this end, let $\hat{u} \in R^{d}, \hat{u} \neq 0$. Observe that

$$
\hat{v}^{T} G \hat{u}=(u, v) \text { for all } \hat{v} \in R^{d},
$$

where

$$
u=\sum_{k=1}^{d} \hat{u}_{k} \alpha_{k} \quad \text { and } \quad v=\sum_{k=1}^{d} \hat{v}_{k} \beta_{k} .
$$

Let $j=\min \left\{i: u \not \equiv 0\right.$ on $\left.\left(x_{i-1}, x_{i}\right)\right\}$. Now suppose that $\int_{x_{j-1}}^{x_{j}} u(x) d x \neq 0$. Then define

$$
v(x)= \begin{cases}\int_{x}^{x_{j}} u(s) d s, & 0 \leqslant x<x_{j}, \\ 0, & x_{j} \leqslant x \leqslant 1 .\end{cases}
$$

Then $v \in \stackrel{\circ}{H}_{\Delta}^{r}(\Omega)$. Hence $v=\Sigma_{k=1}^{d} \hat{v}_{k} \beta_{k}$, for some $\hat{v} \in R^{d}$, and

$$
\begin{aligned}
\hat{v}^{T} G \hat{u} & =(u, v)=\int_{0}^{x_{j}} u(x) v(x) d x \\
& =-\int_{0}^{x_{j}} \frac{\partial v}{\partial x}(x) v(x) d x=\frac{1}{2}|v(0)|^{2}=\frac{1}{2}\left|\int_{x_{j-1}}^{x_{j}} u(s) d s\right|^{2}>0 .
\end{aligned}
$$

Now if $\int_{x_{j-1}}^{x_{j}} u(s) d s=0$, then define

$$
v(x)= \begin{cases}u\left(x_{j-1}+\right)-u\left(x_{j}-\right), & 0 \leqslant x \leqslant x_{j-1}, \\ u(x)-u\left(x_{j}-\right), & x_{j-1}<x<x_{j} \\ 0, & x_{j} \leqslant x \leqslant 1 .\end{cases}
$$

Again $v \in \stackrel{\circ}{H}_{\Delta}^{r}(\Omega)$, and 


$$
\begin{aligned}
\hat{v}^{T} G \hat{u} & =(u, v)=\int_{x_{j-1}}^{x_{j}} u(x)\left[u(x)-u\left(x_{j}-\right)\right] d x \\
& =\int_{x_{j-1}}^{x_{j}}[u(x)]^{2} d x-u\left(x_{j}-\right) \int_{x_{j-1}}^{x_{j}} u(x) d x=\int_{x_{j-1}}^{x_{j}}[u(x)]^{2} d x>0 .
\end{aligned}
$$

Finally, if

$$
G \hat{u}=0 \text { for some } \hat{u} \in R^{d}, \hat{u} \neq 0 \text {, }
$$

then

$$
\hat{v}^{T} G \hat{u}=0 \text { for all } \hat{v} \in R^{d},
$$

which is not possible. Hence $G$ is nonsingular.

The following result is easily obtainable by appropriate local Taylor expansions.

LEMMA 2.2. There exists a constant $0<C^{*}<\infty$, such that for all $u \in H^{s}(\Omega)$,

$$
\underset{\chi \mathrm{INF}}{\mathrm{ING}} \boldsymbol{G}_{\Delta}^{r(\Omega)}\|u-\chi\|_{0} \leqslant C^{*} h^{s}\|u\|_{s}, \quad 0 \leqslant s \leqslant r,
$$

and for all $u \in H^{s}(\Omega) \cap \stackrel{\circ}{H}(\Omega)$,

$$
\underset{\substack{\mathrm{INF} \\ \chi \mathcal{H}_{\Delta}^{r}(\Omega)}}{\|u-\chi\|_{1} \leqslant C^{*} h^{s-1}\|u\|_{s}, \quad 1 \leqslant s \leqslant r+1 .}
$$

$C^{*}$ is independent of $\Delta$ (and $h$ ) but depends on $r$.

The bilinear for $b(\cdot, \cdot)$ defined by $(2.2)$ possesses certain properties on the subspaces $G_{\Delta}^{r}(\Omega)$ and $\stackrel{\circ}{H}_{\Delta}^{r}(\Omega)$ which we now display.

Lemma 2.3. Let $b(\cdot, \cdot)$ be defined by (2.2), then

$$
|b(u, v)| \leqslant\|u\|_{0}\|v\|_{1},
$$

for all $u \in L^{2}(\Omega), v \in H^{1}(\Omega)$, and

$$
\underset{v \in \stackrel{\circ}{H}_{\Delta}^{r}(\Omega) ;\|v\|_{1} \leqslant 1}{\operatorname{SUP}}|b(u, v)| \geqslant \frac{1}{\sqrt{2}}\|u\|_{0},
$$

for all $u \in G_{\Delta}^{r}(\Omega)$.

Proof. Clearly (2.3) follows from Schwarz' inequality. To derive (2.4), let $u \in G_{\Delta}^{r}(\Omega)$. Then define

$$
v(x)=-\int_{1}^{x} u(s) d s, \quad x \in \bar{\Omega} .
$$

Then $v \in \stackrel{\circ}{H}_{\Delta}^{r}(\Omega)$, and by Schwarz' inequality,

$$
\|v\|_{1} \leqslant \sqrt{2}\|u\|_{0} .
$$

From (2.2) and (2.6),

$$
\begin{aligned}
b\left(u, v /\|v\|_{1}\right) & =\|v\|_{1}^{-1}(-u, \partial v / \partial x) \\
& =\|u\|_{0}^{2}\|v\|_{1}^{-1} \geqslant(1 / \sqrt{2})\|u\|_{0} .
\end{aligned}
$$

The result (2.4) now follows.

3. A Projection Theorem. The following theorem will be used in obtaining the error estimates. The result is motivated by the analogous result in [1]. 
THEOREM 3.1. Let $G$ and $H$ be two normed linear spaces with norms $\|\cdot\|_{G}$ and $\|\cdot\|_{H}$, respectively. Let $G$ be a nonempty linear subspace of $G$, and $H$ a nonempty linear subspace of $H$, with

$$
\text { DIMENSION } G=\text { DIMENSION } H<\infty .
$$

Let $b(\cdot, \cdot): G \times H \rightarrow R^{1}$ be a bilinear mapping satisfying

$$
|b(u, v)| \leqslant C_{1}\|u\|_{G}\left\|_{v}\right\|_{H},
$$

for all $u \in G, v \in H$, where $C_{1}<\infty$ is a constant.

There exists a constant $C_{2}>0$ such that

$$
\underset{v \in H ;\|v\|_{H} \leqslant 1}{\mathrm{SUP}}|b(u, v)| \geqslant C_{2}\|u\|_{G} \quad \text { for all } u \in G .
$$

Then if $u_{0} \in G$, there exists a unique $\hat{u}_{0} \in G$ such that

$$
b\left(\hat{u}_{0}, v\right)=b\left(u_{0}, v\right) \text { for all } v \in H,
$$

and

$$
\left\|u_{0}-\hat{u}_{0}\right\|_{G} \leqslant\left(1+C_{1} C_{2}^{-1}\right) \underset{\chi \in G}{\operatorname{INF}}\left\|u_{0}-\chi\right\|_{G} .
$$

Proof. The Eq. (3.3) is equivalent to a system of $d$ linear algebraic equations, the unknowns being the $d$ coefficients of $\hat{u}_{0}$ relative to the chosen basis for $G$, where $d=$ DIMENSION $G=$ DIMENSION $H$.

The condition (3.2) dictates that the associated matrix is nonsingular. Hence $\hat{u}_{0}$ exists uniquely.

The estimate (3.4) may be derived as follows.

Since $G$ is a finite-dimensional linear subspace of the normed linear space $G$, there exists a $\xi \in G$ such that

$$
\left\|u_{0}-\xi\right\|_{G}=\underset{\chi \in G}{\mathrm{INF}}\left\|u_{0}-\chi\right\|_{G} .
$$

Now from (3.2), (3.3) and (3.1),

$$
\begin{aligned}
\left\|\hat{u}_{0}-\xi\right\|_{G} & \leqslant C_{2}^{-1} \underset{v \in H ;\|v\|_{H} \leqslant 1}{\operatorname{SUP}}\left|b\left(\hat{u}_{0}-\xi, v\right)\right| \\
& =C_{2}^{-1} \underset{v \in H ;\|v\|_{H} \leqslant 1}{\operatorname{SUP}}\left|b\left(u_{0}-\xi, v\right)\right| \leqslant C_{1} C_{2}^{-1}\left\|u_{0}-\xi\right\|_{G} .
\end{aligned}
$$

Hence (3.6) gives

$$
\begin{aligned}
\left\|u_{0}-\hat{u}_{0}\right\|_{G} & \leqslant\left\|u_{0}-\xi\right\|_{G}+\left\|\xi-\hat{u}_{0}\right\|_{G} \\
& \leqslant\left(1+C_{1} C_{2}^{-1}\right)\left\|u_{0}-\xi\right\|_{G} \\
& =\left(1+C_{1} C_{2}^{-1}\right) \underset{x \in G}{\operatorname{INF}}\left\|u_{0}-\chi\right\|_{G} .
\end{aligned}
$$

An application of Theorem 3.1 yields:

THEOREM 3.2. Let $u$ be the solution of the boundary value problem (2.1); then for each $\Delta \in \Pi(\Omega)$ there exists a unique mapping $\omega_{\Delta}:[0, T] \rightarrow G_{\Delta}^{r}(\Omega)$ which satisfies 


$$
b\left(\omega_{\Delta}(\cdot, t), v\right)=b(u(\cdot, t), v),
$$

for all $v \in \stackrel{\circ}{H}_{\Delta}^{r}(\Omega)$. Furthermore, if for some integer $k \geqslant 0$,

$$
\partial^{k} u / \partial t^{k} \in L^{p}\left(0, T ; H^{s}(\Omega)\right), \text { then } \partial^{k} \omega_{\Delta} / \partial t^{k} \in L^{p}\left(0, T ; G_{\Delta}^{r}(\Omega)\right),
$$

and there exists a constant $C^{* *}$ independent of $h$ such that

$$
\left\|\left(\partial^{k} / \partial t^{k}\right)\left[u-\omega_{\Delta}\right]\right\|\left\|_{p,-j} \leqslant C^{* *} h^{s+j \|}\right\| \partial^{k} u / \partial t^{k} \|_{p, s}, \quad 0 \leqslant s \leqslant r, 0 \leqslant j \leqslant 1 .
$$

Proof. The existence and uniqueness of an $\omega_{\Delta}:[0, T] \rightarrow G_{\Delta}^{r}(\Omega)$ satisfying (3.7) follows from Lemma 2.3 and Theorem 3.1 by making the identification $G=L^{2}(\Omega)$, $H=\stackrel{\circ}{H}(\Omega), G=G_{\Delta}^{r}(\Omega), H=\stackrel{\circ}{H}_{\Delta}^{r}(\Omega)$. The estimate (3.8) for $j=0$, follows from (3.4) and Lemma 2.2. We now show (3.8) for $j=1$. Set $e=u-\omega_{\Delta}$, and let $\phi \in C^{\infty}(\Omega)$. Now define

$$
\psi(x)=-\int_{1}^{x} \phi(s) d s, \quad x \in \bar{\Omega}
$$

then $\psi \in C^{\infty}(\Omega) \cap \stackrel{\circ}{H}(\Omega)$, and

$$
\|\psi\|_{2} \leqslant \sqrt{2}\|\phi\|_{1} .
$$

Now let $\psi_{\Delta} \in \stackrel{\circ}{H}_{\Delta}^{r}(\Omega)$ be such that

$$
\left\|\psi-\psi_{\Delta}\right\|_{1} \leqslant C^{*} h\|\psi\|_{2} \leqslant \sqrt{2} C^{*} h\left\|_{\phi}\right\|_{1} .
$$

Then from (3.9), (3.7), and (3.11),

hence

$$
\begin{aligned}
(e, \phi) & =b(e, \psi)=b\left(e, \psi-\psi_{\Delta}\right) \\
& \leqslant\|e\|_{0}\left\|\psi-\psi_{h}\right\|_{1} \leqslant \sqrt{2} C^{*} h\left\|_{\phi}\right\|_{1}\|e\|_{0}
\end{aligned}
$$

$$
\|e\|_{-1}=\operatorname{SUP}_{\phi \in C^{\infty}(\Omega) ; \phi \neq 0} \frac{(e, \phi)}{\|\phi\|_{1}} \leqslant \sqrt{2} C^{*} h\left\|_{e}\right\|_{0}
$$

The result now follows.

\section{The Continuous Time Galerkin Approximation.}

THEOREM 4.1. Let $u$ be the solution of the problem (2.1). Then for each $\Delta \in \Pi(\Omega)$, there exists a unique mapping $U_{\Delta}:[0, T] \rightarrow G_{\Delta}^{r}(\Omega)$ satisfying

$$
\left(\left(\partial U_{\Delta} / \partial t\right)(\cdot, t), v\right)+b\left(U_{\Delta}(\cdot, t), v\right)=(f(\cdot, t), v)+g(t) v(0)
$$

for all $v \in \stackrel{\circ}{H}_{\Delta}^{r}(\Omega), t>0$, and

$$
\left(U_{\Delta}(\cdot, 0), v\right)=\left(u_{0}, v\right) \text { for all } v \in G_{\Delta}^{r}(\Omega) .
$$

Furthermore if $u \in L^{\infty}\left(0, T ; H^{r}(\Omega)\right), \partial u / \partial t \in L^{\infty}\left(0, T, H^{r-1}(\Omega)\right)$ and $\partial^{2} u / \partial t^{2} \in$ $L^{2}\left(0, T ; H^{r-1}(\Omega)\right)$, then there exists a constant $C(T)$, independent of $h$, such that

$$
\|\|_{u}-U_{\Delta}\|\|_{\infty, 0} \leqslant C(r) h^{r}\left\{\|u\|_{\infty, r}+\left\|\frac{\partial u}{\partial t}\right\|\left\|_{\infty, r-1}+\right\| \frac{\partial^{2} u}{\partial t^{2}} \|_{2, r-1}\right\}
$$


Proof. That $U_{\Delta}(\cdot, t)=\Sigma_{k=1}^{d} \lambda_{k}(t) \alpha_{k}$ exists uniquely follows from the fact that the Eqs. (4.1). and (4.2) are equivalent to the initial-value problem for the system of $d$ linear ordinary differential equations of the first order,

$$
\begin{aligned}
G(d \vec{\lambda} / d t)(t)+B \vec{\lambda}(t) & =\vec{f}(t), \quad 0<t \leqslant T, \\
A \vec{\lambda}(0) & =\vec{u}_{0},
\end{aligned}
$$

where

$$
\begin{aligned}
\vec{\lambda}(t)= & \left(\lambda_{1}(t), \lambda_{2}(t), \ldots, \lambda_{d}(t)\right)^{T} \\
\vec{f}(t)= & \left(\left(f(\cdot, t), \beta_{1}\right),\left(f(\cdot, t), \beta_{2}\right), \ldots,\left(f(\cdot, t), \beta_{d}\right)\right)^{T} \\
& +\left(g(t) \beta_{1}(0), g(t) \beta_{2}(0), \ldots, g(t) \beta_{d}(0)\right)^{T} \\
\vec{u}_{0}= & \left(\left(u_{0}, \alpha_{1}\right),\left(u_{0}, \alpha_{2}\right), \ldots,\left(u_{0}, \alpha_{d}\right)\right)^{T}
\end{aligned}
$$

and $G, B$ and $A$ are the $d \times d$ matrices $G\left(\left(\alpha_{j}, \beta_{i}\right)\right), B=\left(b\left(\alpha_{j}, \beta_{i}\right)\right)$ and $A=\left(\left(\alpha_{j}, \alpha_{i}\right)\right)$, $i, j=1,2, \ldots, d$. Since $A$ is clearly nonsingular, and from Lemma $2.1, G$ is nonsingular, the system.(4.4) and (4.5) has a unique solution.

The estimate (4.3) may be derived by arguments analogous to those used for standard Galerkin methods for parabolic equations found in [6] .

Let $\omega_{\Delta}$ be defined by (3.7), and let $\eta=u-\omega_{\Delta}, \phi=U_{\Delta}-\omega_{\Delta}$, and $e=u-$ $U_{\Delta}$. Then from (4.1), (3.7) and (2.3), for any $v \in \stackrel{\circ}{H}_{\Delta}^{r}(\Omega)$,

$$
\begin{aligned}
& ((\partial \phi / \partial t)(\cdot, t), v)+b(\phi(\cdot, t), v) \\
& \quad=(f(\cdot, t), v)+g(t) v(0)-\left(\left(\partial \omega_{\Delta} / \partial t\right)(\cdot, t), v\right)-b\left(\omega_{\Delta}(\cdot, t), v\right) \\
& \quad=(f(\cdot, t), v)-b(u(\cdot, t), v)-\left(\left(\partial \omega_{\Delta} / \partial t\right)(\cdot, t), v\right)+g(t) v(0) \\
& \quad=((\partial \eta / \partial t)(\cdot, t), v), \quad t>0 .
\end{aligned}
$$

Now choose

$$
\hat{v}(x, t)=-\int_{1}^{x} \frac{\partial \phi}{\partial t}(s, t) d s, \quad t \geqslant 0, x \in \bar{\Omega}
$$

and define

$$
\psi(x, t)=-\int_{1}^{x} \phi(s, t) d s, \quad t \geqslant 0, x \in \bar{\Omega} .
$$

Then (4.6) becomes

$$
\begin{array}{r}
-((\partial \hat{v} / \partial x)(\cdot, t), \hat{v}(\cdot, t))+(\phi(\cdot, t),(\partial \phi / \partial t)(\cdot, t)) \\
=((\partial \eta / \partial t)(\cdot, t), \hat{v}(\cdot, t)), \quad t>0,
\end{array}
$$

or

$$
\frac{1}{2}|\hat{v}(0, t)|^{2}+\frac{1}{2} \frac{d}{d t}\|\phi(\cdot, t)\|_{0}^{2}=\left(\frac{\partial \eta}{\partial t}(\cdot, t), \hat{v}(\cdot, t)\right)
$$

$$
=\left(\frac{\partial \eta}{\partial t}(\cdot, t), \frac{\partial \psi}{\partial t}(\cdot, t)\right), \quad t>0
$$


Now integrating (4.7) from $t=0$ to $t=\xi \leqslant T$,

$$
\begin{aligned}
\|\phi(\cdot, \xi)\|_{0}^{2} \leqslant\|\phi(\cdot, 0)\|_{0}^{2}+2 \int_{0}^{1} \int_{0}^{\xi} \frac{\partial \eta}{\partial t}(\cdot, t) \frac{\partial \psi}{\partial t}(\cdot, t) d x d t \\
=\|\phi(\cdot, 0)\|_{0}^{2}+2\left(\frac{\partial \eta}{\partial t}(\cdot, \xi), \psi(\cdot, \xi)\right)-2\left(\frac{\partial \eta}{\partial t}(\cdot, 0), \psi(\cdot, 0)\right) \\
\quad-2 \int_{0}^{\xi}\left(\frac{\partial^{2} \eta}{\partial t^{2}}(\cdot, t), \psi(\cdot, t)\right) d t \\
\leqslant\|\phi(\cdot, 0)\|_{0}^{2}+4\left\|\frac{\partial \eta}{\partial t}\right\|_{\infty,-1}\|\psi \psi\|_{\infty, 1} \\
\quad+2 \sqrt{T}\left\|\frac{\partial^{2} \eta}{\partial t^{2}}\right\|_{2,-1}\|\psi \psi\|_{\infty, 1} .
\end{aligned}
$$

Now from (4.7),

$$
\|\|\|\|_{\infty, 1} \leqslant \sqrt{2}\|\|_{\phi} \|_{\infty, 0}
$$

Hence, using (4.10) in (4.9),

$$
\begin{aligned}
\|\phi(\cdot, \xi)\|_{0}^{2} \leqslant & \|\phi(\cdot, 0)\|_{0}^{2}+\left\{4 \| \frac{\left\|\frac{\partial \eta}{\partial t} \mid\right\|_{\infty,-1}+2 \sqrt{T}\left\|\frac{\partial^{2} \eta}{\partial t^{2}}\right\|_{2,-1}}{\}^{2}}\right. \\
& +\frac{1}{2}\|\|_{\phi} \|_{\infty, 0}^{2}, \quad 0 \leqslant \xi \leqslant T .
\end{aligned}
$$

On taking the supremum over $\xi$ in (4.11), we get, on using (3.8),

$$
\begin{aligned}
&\|\phi\|_{\infty, 0} \leqslant \sqrt{2}\left\{\|\phi(\cdot, 0)\|_{0}+4\left\|\frac{\partial \eta}{\partial t}\right\|\left\|_{\infty,-1}+2 \sqrt{T}\right\| \frac{\partial^{2} \eta}{\partial t^{2}}\|\|_{2,-1}\right\}\left.+2 \sqrt{T}\left\|\frac{\partial^{2} \eta}{\partial t^{2}}\right\|_{2,-1}\right\} \\
& \leqslant \sqrt{2}\left\{\left\|U_{\Delta}(\cdot, 0)-u_{0}\right\|_{0}+\left\|u_{0}-\omega_{\Delta}(\cdot, 0)\right\|_{0}+4\left\|\frac{\partial \eta}{\partial t}\right\| \|_{\infty,-1}\right. \\
& \leqslant \sqrt{2} C^{* *} h^{r}\left\{2\left\|u_{0}\right\|_{r}+4\left\|\frac{\partial u}{\partial t}\right\|\left\|_{\infty, r-1}+2 \sqrt{T}\right\| \frac{\partial^{2} u}{\partial t^{2}} \|\left.\right|_{2, r-1}\right\} .
\end{aligned}
$$

Hence finally again by (3.8),

$$
\begin{aligned}
\|l\|_{\infty, 0} & \leqslant\|\phi\|_{\infty, 0}+\|m\|_{\infty, 0} \\
& \leqslant C(T) h^{r}\left\{\|\| u\left\|_{\infty, r}+\right\| \frac{\partial u}{\partial t}\|\|_{\infty, r-1}+\left.\left\|\frac{\partial^{2} u}{\partial t^{2}}\right\|\right|_{2, r-1}\right\},
\end{aligned}
$$

where $C=C^{* *} \operatorname{MAX}\{4,2 \sqrt{T}\} \sqrt{2}$. This concludes the proof.

We remark that the definition of $U_{\Delta}(\cdot, 0)$ in $G_{\Delta}^{r}(\Omega)$ is arbitrary up to being an optimal $L^{2}$ approximation to $u_{0}$. (4.2) defines one such choice. 
5. A Discrete Time Galerkin Method. A Crank-Nicolson type Galerkin method based on the weak formulation (2.3) is analyzed here. Analogous schemes for parabolic equations have been proposed in [2] and [3].

Let $T=J \tau$ for some integer $J \geqslant 1$. For a sequence $\left\{V_{n}\right\}_{n=0}^{J} \subset L^{2}(\Omega)$, we define

$$
\begin{aligned}
\partial_{\tau} V^{n} & =\tau^{-1}\left[V^{n+1}-V^{n}\right], \\
V^{n+1 / 2} & =1 / 2\left[V^{n+1}+V^{n}\right], \quad n=0,1, \ldots, J-1, \\
\partial_{\tau}^{2} V^{n} & =\tau^{-2}\left[V^{n+1}-2 V^{n}+V^{n-1}\right], \quad n=1,2, \ldots, J-1,
\end{aligned}
$$

and

$$
\|V\|\left\|_{\infty, 0}=\max _{0 \leqslant n \leqslant J}\right\| V^{n} \|_{0} .
$$

For a mapping $V: C[0, T] \rightarrow L^{2}(\Omega)$, we shall write

$$
V^{n}=V(\cdot, n \tau), \quad n=0,1, \ldots, J .
$$

Throughout the rest of the paper, $C$ will denote a generic constant, not necessarily the same in any two places.

The following result defines a fully discrete Galerkin approximation, and gives the error estimates.

THEOREM 5.1. Let $u$ be the solution of the boundary value problem (2.1), then there exists a unique sequence $\left\{U_{\Delta}^{n}\right\}_{n=0}^{J} \subset G_{\Delta}^{r}(\Omega)$ which satisfies

$$
\left(U_{\Delta}^{0}, \chi\right)=\left(u_{0}, \chi\right) \text { for all } \chi \in G_{\Delta}^{r}(\Omega)
$$

and

$$
\left(\partial_{\tau} U_{\Delta}^{n}, \chi\right)+b\left(U_{\Delta}^{n+1 / 2}, \chi\right)=\left(f^{n+1 / 2}, \chi\right)+g^{n+1 / 2} \chi(0)
$$

for all $\chi \in \stackrel{\circ}{H}_{\Delta}^{r}(\Omega), n=0,1, \ldots, J-1$.

Furthermore if $u \in L^{\infty}\left(0, T ; H^{r}(\Omega)\right), \partial u / \partial t \in L^{\infty}\left(0, T ; H^{r-1}(\Omega)\right), \partial^{2} u / \partial t^{2} \in$ $L^{2}\left(0, T ; H^{r-1}(\Omega)\right), \partial^{3} u / \partial t^{3} \in L^{\infty}\left(0, T ; H^{-1}(\Omega)\right)$, and $\partial^{4} u / \partial t^{4} \in L^{2}\left(0, T ; H^{-1}(\Omega)\right)$, then there exists a constant $C=C(u, T)$ such that

$$
\max _{0 \leqslant n \leqslant J}\left\|u(\cdot, n \tau)-U_{\Delta}^{n}\right\|_{0} \leqslant C\left\{h^{r}+\tau^{2}\right\} .
$$

Proof. Clearly $U_{\Delta}^{0} \in G_{\Delta}^{r}(\Omega)$ is uniquely defined. Now for $n=0,1, \ldots, J-1$, $U_{\Delta}^{n+1}$ satisfies

$$
\left[U_{\Delta}^{n+1}, \chi\right]=F_{n} \chi \quad \text { for all } \chi \in \stackrel{\circ}{H}_{\Delta}^{r}(\Omega),
$$

where $[\cdot, \cdot]: G_{\Delta}^{r}(\Omega) \times \stackrel{\circ}{H}_{\Delta}^{r}(\Omega) \rightarrow R^{1}$ is the bilinear form given by

$$
[U, \chi]=(U, \chi)-(\tau / 2)(U, \partial \chi / \partial x)
$$

and $F_{n}: \stackrel{\circ}{H}_{\Delta}^{r}(\Omega) \rightarrow R^{1}$ is the functional given by

$$
F_{n} \chi=\left(U_{\Delta}^{n}, \chi\right)+(\tau / 2)\left(U_{\Delta}^{n}, \partial \chi / \partial x\right)+\left(f^{n+1 / 2}, \chi\right)+g^{n+1 / 2} \chi(0) .
$$

Since if $U \in G_{\Delta}^{r}(\Omega)$ and 


$$
V(x)=-\int_{1}^{x} U(s) d s, \quad \text { then } V \in \stackrel{\circ}{H}^{r}(\Omega)
$$

and

$$
[U, V]=(\tau / 2)\|U\|_{0}^{2}+1 / 2|V(0)|^{2} \geqslant(\tau / 2)\|U\|_{0}^{2} .
$$

Hence the matrix $\left(\left[\alpha_{j}, \beta_{i}\right]\right)_{i, j=1,2, \ldots, d}$ is nonsingular, and so $U_{\Delta}^{n}$ exists uniquely, $n=0,1, \ldots, J$.

We now derive the estimate (5.3). Again let $\omega_{\Delta} \in G_{\Delta}^{r}(\Omega)$ be defined by (3.7), and set $\eta=u-\omega_{\Delta}, \phi^{n}=U^{n}-\omega_{\Delta}^{n}$ and $e^{n}=u^{n}-U_{\Delta}^{n}$.

From (2.2) it follows that

$$
\left(\partial_{\tau} u^{n}, \chi\right)+b\left(u^{n+1 / 2}, \chi\right)=\left(f^{n+1 / 2}+\rho^{n}, \chi\right)+g^{n+1 / 2} \chi(0)
$$

for all $\chi \in \stackrel{\circ}{H}(\Omega)$, where

$$
\rho^{n}=\partial_{\tau} u^{n}-\partial u^{n+1 / 2} / \partial t, \quad n=0,1, \ldots, J-1 .
$$

Hence from (5.2), (3.7) and (5.4), for $\chi \in \stackrel{\circ}{H}^{r}(\Omega)$,

$$
\begin{aligned}
\left(\partial_{\tau} \phi^{n}, \chi\right)+b\left(\phi^{n+1 / 2}, \chi\right) & \\
= & \left(f^{n+1 / 2}, \chi\right)+g^{n+1 / 2} \chi(0)-\left(\partial_{\tau} \omega^{n}, \chi\right)-b\left(\omega_{\Delta}^{n+1 / 2}, \chi\right) \\
= & \left(f^{n+1 / 2}, \chi\right)-b\left(u^{n+1 / 2}, \chi\right)-\left(\partial_{\tau} \omega^{n}, \chi\right)+g^{n+1 / 2} \chi(0) \\
& =\left(\partial_{\tau} \eta^{n}-\rho^{n}, \chi\right), \quad n=0,1, \ldots, J-1 .
\end{aligned}
$$

We now make the choice

$$
\hat{\chi}^{n}(x)=-\int_{1}^{x} \partial_{\tau} \phi^{n}(s) d s, \quad x \in \bar{\Omega} .
$$

Then $\left\{\hat{\chi}^{n}\right\}_{n=0}^{J} \subset \stackrel{\circ}{H}_{\Delta}^{r}(\Omega)$.

Using (5.7) and (5.6),

$$
-\left(\frac{\partial \hat{\chi}^{n}}{\partial x}, \hat{\chi}^{n}\right)+\left(\phi^{n+1 / 2}, \partial_{\tau} \phi^{n}\right)=\left(\partial_{\tau} \eta^{n}-\rho^{n}, \hat{\chi}^{n}\right)
$$

or

$$
\begin{aligned}
1 / 2\left|\hat{\chi}^{n}(0)\right|^{2} & +1 / 2 \tau^{-1}\left(\left\|\phi^{n+1}\right\|_{0}^{2}-\left\|\phi^{n}\right\|_{0}^{2}\right) \\
= & \left(\partial_{\tau} \eta^{n}-\rho^{n}, \hat{\chi}^{n}\right), \quad n=0,1, \ldots, J-1 .
\end{aligned}
$$

Now define $\psi^{n}(x)=\int_{0}^{x}\left[\partial_{\tau} \eta^{n}(s)-\rho^{n}(s)\right] d s$. Then since $\psi^{n}(0)=0$, it can be easily verified that

$$
\left\|\psi^{n}\right\|_{0} \leqslant C\left\|\partial \psi^{n} / \partial x\right\|_{-1}=\left\|\partial_{\tau} \eta^{n}-\rho^{n}\right\|_{-1}
$$

also

$$
\left(\partial_{\tau} \eta^{n}-\rho^{n}, \hat{\chi}^{n}\right)=\left(\partial \psi^{n} / \partial x, \hat{\chi}^{n}\right)=-\left(\psi^{n}, \partial \hat{\chi}^{n} / \partial x\right)=\left(\psi^{n}, \partial_{\tau} \phi^{n}\right)
$$

Hence if we substitute (5.10) in (5.8), and sum for $n=0$ to $n=l-1$, for some $1 \leqslant l \leqslant J$, then 


$$
\begin{aligned}
\left\|\phi^{l}\right\|_{0}^{2} & \leqslant\left\|\phi^{0}\right\|_{0}^{2}+2 \tau \sum_{n=0}^{l-1}\left(\psi^{n}, \partial_{\tau} \phi^{n}\right) \\
& =\left\|\phi^{0}\right\|_{0}^{2}+2\left[\left(\phi^{l}, \psi^{l-1}\right)-\left(\phi^{0}, \psi^{0}\right)\right]+2 \tau \sum_{n=1}^{l-1}\left(\phi^{n}, \partial_{\tau} \psi^{n-1}\right) .
\end{aligned}
$$

From (5.5) one may prove that

$$
\|\rho\|\left\|_{\infty,-1} \leqslant C \tau^{2}\right\| \partial^{3} u / \partial t^{3}\|\|_{\infty,-1},
$$

and so from (5.9), (5.5) and (3.8), for $0 \leqslant n \leqslant J-1$,

$$
\begin{aligned}
\left\|\psi^{n}\right\|_{0} & \leqslant C\left\|\partial_{\tau} \eta^{n}\right\|_{-1}+C\left\|\rho^{n}\right\|_{-1} \\
& \leqslant C\left\{h^{r}\left\|\frac{\partial u}{\partial t}\right\|\left\|_{\infty, r-1}+\tau^{2}\right\| \frac{\partial^{3} u}{\partial t^{3}}\|\|_{\infty,-1}\right\}
\end{aligned}
$$

Also, since

$$
\partial_{\tau} \psi^{n-1}=\int_{0}^{x}\left[\partial_{\tau}^{2} \eta^{n}(s)-\partial_{\tau} \rho^{n-1}(s)\right] d s,
$$

it follows that

$$
\left\|\partial_{\tau} \psi^{n-1}\right\|_{0} \leqslant C\left\|\partial_{\tau}^{2} \eta^{n}-\partial_{\tau} \rho^{n-1}\right\|_{-1} .
$$

Using (5.14) and (3.8), we obtain

$$
\begin{aligned}
\tau \sum_{n=1}^{l-1}\left\|\partial_{\tau} \psi^{n-1}\right\|_{0}^{2} & \leqslant C\left\{\left.\left\|\frac{\partial^{2} \eta}{\partial t^{2}}\right\|\right|_{2,-1} ^{2}+\left.\tau^{4}\left\|\frac{\partial^{4} u}{\partial t^{4}}\right\|\right|_{2,-1} ^{2}\right\} \\
& \leqslant C\left\{h^{r}\left\|\frac{\partial^{2} u}{\partial t^{2}} \mid\right\|_{2, r-1}+\tau^{2}\left\|\frac{\partial^{4} u}{\partial t^{4}}\right\|_{2,-1}\right\}^{2} .
\end{aligned}
$$

Returning to (5.11) with the estimates (5.13) and (5.15),

$$
\begin{aligned}
\left\|\phi^{l}\right\|_{0}^{2} \leqslant & \left\|\phi^{0}\right\|_{0}^{2}+4\|\|_{\phi}\left\|_{\infty, 0}\right\| \psi \psi\|\|_{\infty, 0} \\
& +2 \tau\left(\sum_{n=1}^{l-1}\left\|\phi^{n}\right\|_{0}^{2}\right)^{1 / 2}\left(\sum_{n=1}^{l-1}\left\|\partial_{\tau} \psi^{n-1}\right\|_{0}^{2}\right)^{1 / 2} \\
\leqslant & \left\|\phi^{0}\right\|_{0}^{2}+\frac{1}{4}\|\|_{\phi}\left\|_{\infty, 0}+4^{3}\right\| \psi\|\|_{\infty, 0}^{2} \\
& +\frac{1}{4}\|\|_{\phi}\left\|_{\infty, 0}^{2}+16 T^{2} \tau \sum_{n=1}^{l-1}\right\| \partial_{\tau} \psi^{n-1} \|_{0}^{2} \\
\leqslant & \left\|\phi^{0}\right\|_{0}^{2}+\frac{1}{2}\|\|_{\phi} \|_{\infty, 0}+C(T)\left\{h^{r}\left[\left\|\frac{\partial u}{\partial t}\right\|\left\|_{\infty, r-1}+\right\| \frac{\partial^{2} u}{\partial t^{2}}\|\|_{2, r-1}\right]\right. \\
& \left.+\tau^{2}\left[\left\|\frac{\partial^{3} u}{\partial t^{3}}\right\|\left\|_{\infty,-1}+\right\| \frac{\partial^{4} u}{\partial t^{4}}\|\|_{2,-1}\right]\right\}^{2} .
\end{aligned}
$$


Hence taking the maximum in (5.16),

$$
\begin{array}{r}
\|\phi\|_{\infty, 0} \leqslant C(T)\left\{\left\|\phi^{0}\right\|_{0}+h^{r}\left[\left\|\frac{\partial u}{\partial t}\right\|\left\|_{\infty, r-1}+\right\| \frac{\partial^{2} u}{\partial t^{2}} \|_{2, r-1}\right]\right. \\
\left.+\tau^{2}\left[\left\|\frac{\partial^{3} u}{\partial t^{3}}\right\|\left\|_{\infty,-1}+\right\| \frac{\partial^{4} u}{\partial t^{4}}\|\|_{2,-1}\right]\right\} .
\end{array}
$$

Now from (3.8)

$$
\left\|\phi^{0}\right\|_{0} \leqslant\left\|e^{0}\right\|_{0}+\left\|\eta^{0}\right\|_{0} \leqslant C h^{r}\left\|_{u_{0}}\right\|_{r}
$$

hence

$$
\begin{aligned}
& \|\| l\left\|_{\infty} \leqslant\right\|\left\|_{\eta}\right\|_{\infty}+\|\| \phi\|\|_{\infty} \\
& \leqslant C\left\{h^{r}\left[\|u\|_{\infty, r}+\left\|\frac{\partial u}{\partial t}\right\|\left\|_{\infty, r-1}+\right\| \frac{\partial^{2} u}{\partial t^{2}}\|\|_{2, r-1}\right]\right. \\
& \left.+\tau^{2}\left[\left\|\frac{\partial^{3} u}{\partial t^{3}}\right\|\left\|_{\infty,-1}+\right\| \frac{\partial^{4} u}{\partial t^{4}} \|_{2,-1}\right]\right\} \text {. }
\end{aligned}
$$

The result (5.3) now follows.

The Aiken Computation Laboratory

Division of Engineering and Applied Physics

Harvard University

Cambridge, Massachusetts 02138

1. I. BABUSKKA, "Error-bounds for finite element method," Numer. Math., v. 16, 1970/71, pp. 322-333. MR 44 \#6166.

2. J. H. BRAMBLE \& V. THOMÉE, Discrete Time Galerkin Methods for a Parabolic Boundary Value Problem, MRC Tech. Report \#1240, University of Wisconsin, 1972.

3. J. DOUGLAS, JR. \& T. DUPONT, "Galerkin methods for parabolic equations," SIAM J. Numer. Anal., v. 7, 1970, pp. 575-626. MR 43 \#2863.

4. J. L. LIONS \& E. MAGENES, Non-Homogeneous Boundary Value Problems and Applications. Vol. I, Springer-Verlag, New York, 1972.

5. H. H. RACHFORD, JR. \& M. F. WHEELER, " $H^{-1}$ Galerkin procedure for the two point boundary value problem," Mathematical Aspects of the Finite Element Method, C. DeBoor (Editor), Academic Press, New York, 1974.

6. M. SCHECTER. "On $L^{p}$ estimates and regularity. II," Math. Scand., v. 13, 1963, pp. 4769. MR 32 \#6052.

7. M. F. WHEELER, "A priori $L^{2}$ error estimates for Galerkin approximations to parabolic partial differential equations," SIAM J. Numer. Anal., v. 10, 1973, pp. 723-759. 